\title{
On the nature of striped phases: Striped phases as a stage of "melting" of 2D crystals
}

\author{
Volodymyr Derzhko ${ }^{1}$, Janusz Jȩdrzejewski ${ }^{1 *}$ and Taras Krokhmalskiii ${ }^{2}$ \\ ${ }^{1}$ Institute of Theoretical Physics, University of Wrocław, \\ pl. Maksa Borna 9, 50-204 Wrocław, Poland \\ 2 Institute for Condensed Matter Physics, \\ 1 Svientsitskii Str., Lviv-11, 79011, Ukraine
}

July 1, 2018

\begin{abstract}
We discuss striped phases as a state of matter intermediate between two extreme states: a crystalline state and a segregated state. We argue that this state is very sensitive to weak interactions, compared to those stabilizing a crystalline state, and to anisotropies. Moreover, under suitable conditions a 2D system in a striped phase decouples into (quasi) 1D chains. These observations are based on results of our studies of an extension of a microscopic quantum model of crystallization, proposed originally by Kennedy and Lieb.
\end{abstract}

\section{Introduction}

Striped phases, specific phases having quasi-one-dimensional structure, are ubiquitous. They have been observed in numerous experiments carried out in a variety of systems, among which there are physisorbed monolayers on metallic surfaces [1] or ultrathin magnetic garnet films [2, 3]. They have also been found in theoretical analyses, carried out by analytic or numerical methods, of various quantum and classical model systems. In the class of quantum models, one can find results pertaining to the Hubbard model [4, 5, 6], $t-J$ model [7, 8], $X Y$ model [9], spinless fermion model [10, 11], spinless Falicov-Kimball model [12, 13]. It has been argued in a number of works that some essential physics of systems displaying striped phases can be grasped by a kind of coarse-grained descriptions that result in classical lattice-gas models (Ising-like models or $O(n)$ models) with competing two-body interactions. Typically, in these models short-range attractive (ferromagnetic) interactions compete with long-range repulsive ones [14, 15, 16, 17, 18, 19. Interestingly, despite the difficulty of determining ground states of such systems, in recent years a few rigorous results have been published [20, 21].

Perhaps the most notable striped phases are those observed in doped layered perovskites, which under suitable conditions become high-temperature superconductors [22, 23]. The

${ }^{*}$ Corresponding author: J. Jȩdrzejewski, phone: +48 71 3759415, fax: +48 71 3214454, e-mail: jjed@ift.uni.wroc.pl 
nature of striped phases in these materials, in particular their competition with a superconducting state, is still vigorously debated.

The purpose of this paper is threefold. Firstly, we argue that the problem of the formation of striped phases falls naturally into the context of an extended crystallization problem, that is not only the existence of crystalline phases is addressed but also the process of their deterioration into a segregated state (described below) as a result of weakening of the forces stabilizing a crystalline state. A crystalline phase and a segregated one might be thought of as two extreme states of matter. Presumably, striped phases appear in this process as the last but one stage of a sequence of phase transformations that starts with a crystalline state and ends with a segregated one.

Secondly, we point out that the stability of striped phases might be rather fragile. Very weak interactions, in the hierarchy of the interactions present in the system under consideration, can change the character of the striped pattern or destabilize completely striped phases. Moreover, even a weak anisotropy of the system chooses the direction of stripes in stable striped phases.

Thirdly, the appearance of striped phases might signal a dramatic destruction of correlations in direction of stripes. An anisotropy of the system favors this effect. Specifically, we show that when our system is in an axial-stripe phase, zero-temperature electron correlations (given by ground-state one-body reduced density matrix) along the stripes vanish. We make an attempt to estimate the range of temperatures in which this property holds approximately. Consequently, before a 2D crystal deteriorates completely (to a state of segregation), it transforms effectively into a quasi one-dimensional structure, destroying some of existing 2D long-range orders.

Our main conclusions and suggestions are based on a rigorous analysis of an extension of a microscopic quantum model of crystallization, which was proposed twenty two years ago by Kennedy and Lieb [24]. Some additional observations are based on numerical calculations. In brief, the Kennedy and Lieb model of crystallization is a two-component fermionic system on a lattice that consists of heavy immobile ions and light hopping electrons, and the sole interaction in this system is an on-site electron-ion interaction representing a screened Coulomb interaction.

The paper is organized as follows. In the next section we introduce our model of crystallization. Then, in Section 3, we present some of the ground-state phase diagrams of our system, discuss possible scenarios of deterioration of a checkerboard-like 2D crystal, emphasizing the role of striped phases and the effect of an anisotropy of electron hopping. After that, in Section 4, we focus our attention on axial-stripe phases, specifically on electron correlations in such phases. Finally, in Section 5, we summarize our results. Spectral properties of axial-stripe phases are presented in Appendix.

\section{The model of crystallization}

The model of crystallization proposed by Kennedy and Lieb 24] is composed of two fermionic subsystems: light hopping electrons and heavy immobile ions. The electrons are represented by spinless fermions (spin does not play any role in our considerations), described by creation and annihilation operators of an electron at a state localized at site $x$ of the underlying lattice, $c_{x}^{+}, c_{x}$, respectively, satisfying the canonical anticommutation relations. The ions are described by collections of pseudo-spins $\left\{s_{x}\right\}_{x \in \Lambda}$, called the ion configurations; $s_{x}=1$ if the site $x$ is occupied by an ion and $s_{x}=-1$ if it is empty. The pseudo-spins commute with the creation and annihilation operators of electrons. 
There is neither a direct interaction between mobile electrons nor between immobile ions. The electrons energy is due to hopping (typically a nearest-neighbor (n.n.) hopping is assumed) with $t$ being the n.n. hopping intensity (without any loss of generality we can fix the sign of $t, t>0$ ), and due to a screened Coulomb interaction with the immobile ions, whose strength is controlled by the coupling constant $U>0$ (concerning the sign see a comment in the text below). The Hamiltonian of the system reads:

$$
H_{F K}=t \sum_{\langle x, y\rangle_{1}}\left(c_{x}^{+} c_{y}+c_{y}^{+} c_{x}\right)+U \sum_{x}\left(c_{x}^{+} c_{x}-\frac{1}{2}\right) s_{x}
$$

where $\langle x, y\rangle_{1}$ means that the sites $x, y$ constitute a pair of n.n. sites. In the sequel, we shall limit our considerations only to the case in which the underlying lattice is a square lattice.

It is worth to emphasize here that although the ions are not moving due to the dynamics of the system, their configurations are not frozen or random. On calculating the canonical partition function, one takes a trace over electronic degrees of freedom and sums over all configurations of the ions. It is this sampling of possible configurations that produces correlations between ions, which in turn may lead to a crystalline arrangement of the ions.

The Hamiltonian $H_{F K}$ is widely known as the Hamiltonian of the spinless Falicov-Kimball model, a simplified version of the Hamiltonian put forward for describing electronic subsystems of some solids in [25]. To the best of our knowledge, the spinless-fermion FalicovKimball model is the unique system of interacting fermions, for which the existence of longrange orders (periodic phases) and phase separations have been proved. The majority of rigorous results refers to the ground states of the model and holds only in the strong-coupling regime, i.e. for sufficiently small $|t / U|$, and for particle densities satisfying specific conditions (some more details and comments can be found below). Interestingly, the first proofs of the existence of a periodic phase, which in $2 \mathrm{D}$ is the so called checkerboard phase with the electron and ion densities equal 1/2, hold for any $U$ : at zero temperature [26, 24] and for sufficiently low temperatures [24]. It is the checkerboard phase that in our considerations is identified with an initial crystal, which is given a possibility to deteriorate. A review of results pertaining to ground-state or low-temperature phase diagrams and an extensive list of relevant references can be found in [27, 28]).

According to the state of art, a quite general analysis of ground-state phase diagrams, that made possible proving the existence of numerous periodic phases and mixtures (phase separated states) of such phases, is feasible only in the mentioned above strong-coupling regime, and when the densities of the ions and electrons, $\rho_{i}$ and $\rho_{e}$, respectively, satisfy a specific condition. Either it is the condition of neutrality, $\rho_{i}=\rho_{e}$, if the electron-ion interaction is attractive, or it is the condition of half-filling, $\rho_{i}+\rho_{e}=1$, if that interaction is repulsive. The two cases are related by a unitary transformation: a hole-particle transformation (for definiteness, we assume in our considerations that the electron-ion interaction is repulsive and the system is half-filled). In the specified above regime, it is possible to derive explicitly, in the form of a convergent power series with respect to the small parameter $|t / U|$, an effective interaction between the ions [29, 30, 31, 32]. The components of this expansion constitute many-body finite-range lattice-gas interactions, where the number of interacting bodies and the range of interaction grow without bound with the order of those components. Then, it is possible to construct rigorously the ground-state phase diagram of the effective interaction truncated at certain order, and after that derive information on the groundstate phase diagram of the complete quantum system under consideration [29, 30, 31, 32. The ground-state results obtained in the described way can be extended to sufficiently low temperatures [32]. 
The emerging phase diagram is very reach; besides a few periodic phases [31, 33, 34, 35] and various phase-separated states [33, 34, that can be determined rigorously, it contains most probably infinitely many periodic phases and mixtures of periodic phases (see [36, 37, 38, 39] for the results obtained by means of the method of restricted phase diagrams). However at half-filling and large $U$, it does not contain any segregated phases, that is thermodynamic mixtures of the completely filled with ions phase $\left(\rho_{i}=1\right)$ and the ion void phase $\left(\rho_{i}=0\right)$ with suitable electron densities. This is in agreement with rigorous results of [40, 41, 42], which state that a segregated phase is stable for any total density away from half-filling if $U$ is large enough. Approximate results of [36, 37, 38] suggest that whatever $U>0$, there is no segregated phases at half-filling.

We insist, however, that a crystalline state, like the half-filled checkerboard phase, should have a possibility to deteriorate into the half-filled segregated phase, considered as a final stage of a deterioration of a crystal, for any $U$. The standard Falicov-Kimball model cannot encompass such a "process": there is neither the stable half-filled segregated phase nor a parameter to drive such a process. The reason for which half-filled segregated phases are missing in the standard spinless Falicov-Kimball model, in the strong-coupling regime, might be attributed to the fact that the leading interaction term (whose order is $O\left(|t / U|^{2}\right)$ ) of the effective interaction expansion is a n.n. repulsive lattice gas (or in terms of pseudo-spins - an Ising antiferromagnet), irrespectively of the sign of $U$. In order to stabilize the halffilled segregated phase and to provide the standard model with a suitable control parameter, we extend it by adding a small (second order as compared to the electron-ion interaction) short-range attractive interaction between the ions. For simplicity, we choose its range to be limited to the distance between next-nearest-neighbor (n.n.n.) sites. The physical source of this interaction might be identified as van der Waals forces, which despite their weakness are known to play an important role in stability of crystals. Therefore, we extend the standard spinless Falicov-Kimball model by adding to the Hamiltonian $H_{F K}$ the term $H_{v d W}$,

$$
H_{v d W}=\frac{W}{8} \sum_{\langle x, y\rangle_{1}} s_{x} s_{y}-\frac{\tilde{\varepsilon}}{16} \sum_{\langle x, y\rangle_{2}} s_{x} s_{y}
$$

with

$$
W=-2 t^{2}+t^{4} \omega, \quad \tilde{\varepsilon}=t^{4} \varepsilon
$$

where $\langle x, y\rangle_{2}$ stands for a pair of n.n.n. sites. The parameters $\omega$ and $\varepsilon$, which vary the strength of n.n. and n.n.n. couplings in $H_{v d W}$, respectively, are the control parameters of the phase diagrams discussed in the sequel. The specific form of $W$, given in (3), and the coefficients that stand by the parameters $W$ and $\tilde{\varepsilon}$ in (2) stem from our analysis of the ground-state phase diagram of the 2nd order effective interaction (see [13 for details). In those diagrams, $\omega=\varepsilon=0$ is the point of coexistence of the checkerboard phase and the half-filled segregated phase. Despite the weakness of the n.n.n. term in $H_{v d W}$, it will become clear in the discussion of phase diagrams that follows, that extending the range of $H_{v d W}$ beyond n.n. enables us to make interesting observations pertaining to a transition from a crystalline state to a segregated one.

To take into account important effects of anisotropy of electron hopping, we differentiate between hopping in the vertical and horizontal direction by introducing the corresponding hopping intensities $t_{v}>0$ and $t_{h}>0$, and the anisotropy parameter $\gamma$ such that $t_{v}=\sqrt{\gamma} t_{h}$, with $0 \leq \gamma \leq 1$ [43] (this somewhat unusual definition of the anisotropy parameter is dictated by the simplicity of the expression for the effective interaction). Then, in the case of the 
hole-particle invariant system, the effective interaction (in the units of $U$ ) between the ions, up to order 4, reads:

$$
\begin{aligned}
& {\left[\frac{t^{2}}{4}-\frac{3 t^{4}}{16}-\frac{3}{8} \gamma t^{4}+\frac{W}{8}\right] \sum_{\langle x, y\rangle_{1, h}} s_{x} s_{y}+} \\
& {\left[\gamma \frac{t^{2}}{4}-\frac{3}{8} \gamma t^{4}-\gamma^{2} \frac{3 t^{4}}{16}+\frac{W}{8}\right] \sum_{\langle x, y\rangle_{1, v}} s_{x} s_{y}+} \\
& {\left[\gamma \frac{3 t^{4}}{16}-\frac{\tilde{\varepsilon}}{16}\right] \sum_{\langle x, y\rangle_{2}} s_{x} s_{y}+\frac{t^{4}}{8} \sum_{\langle x, y\rangle_{3, h}} s_{x} s_{y}+} \\
& \gamma^{2} \frac{t^{4}}{8} \sum_{\langle x, y\rangle_{3, v}} s_{x} s_{y}+\gamma \frac{t^{4}}{16} \sum_{P}\left(1+5 s_{P}\right),
\end{aligned}
$$

where $t=t_{h} / U,\langle x, y\rangle_{n, h}\left(\langle x, y\rangle_{n, v}\right)$ stands for pairs of $n$ th-order nearest-neighbor sites (n.n.1st order, etc) in horizontal (vertical) directions, and $s_{P}$ denotes a product of four spins whose sites constitute an elementary square, $P$, on the lattice.

Since we are working with a truncated effective interaction, we have to assign an order to the deviation of the anisotropy parameter $\gamma$ from the value 1 (the isotropic case). For this purpose we define the anisotropy order, $a$, and the new anisotropy parameter, $\beta_{a}: \gamma=$ $1-\beta_{a} t^{a}, \beta_{a}>0$. In order to analyse anisotropy effects with the effective interaction truncated at certain order, the anisotropy order has to be suitably adjusted. In our work, we analyze the effective interaction truncated at 4 th order, hence the weakest admissible deviation from the isotropic case corresponds to $a=2$.

\section{Ground-state phase diagrams}

The ground-state phase diagrams constructed with effective interaction (44) are shown in Fig. 1, The essential point is that they can also be thought of as ground-state phase diagrams of the full quantum system, provided that the coexistence lines of the phases are interpreted as strips whose width is of the order $O\left(|t / U|^{2}\right)$. Inside these strips, we cannot claim the stability of any phase, but we can exclude the stability of some phases. Studies carried out by approximate methods suggest that these strips may accommodate infinitely many phases [36, 37, 38]. To unveil these phases rigorously, it is necessary to construct phase diagrams according to the effective interactions truncated at higher orders, but this task becomes quickly hardly feasible.

Because of the hole-particle invariance, in all the phases of the phase diagrams shown in Fig. 1 the densities of the ions and the electrons are 1/2; off the hole-particle symmetry case, many other phases of different than $1 / 2$ densities can be proven to be stable [43]. Suppose that initially our system is in a crystalline checkerboard phase, $\mathcal{S}_{c b}$, Fig. 1. Then, by decreasing the "van der Waals interaction" coupling $\omega$, the system is always driven out

of the crystalline state. However, the terminal state and the way it is attained depend strongly on variation of the tiny coupling controlled by $\varepsilon$. It is worth to note here that the complete n.n.n., 4th order, effective interaction consists of a contribution from $H_{F K}$ and from $H_{v d W}$; it is attractive if $\varepsilon>3$, irrespectively of the value of the electron hopping anisotropy controlled by $\beta_{2}$, and then it stabilizes the checkerboard phase. Concerning a terminal state, one can observe that if $\varepsilon$ does not decrease (while $\omega$ decreases), then the terminal state is always the segregated phase, which is, in this case, a fifty-fifty mixture of the uniform 

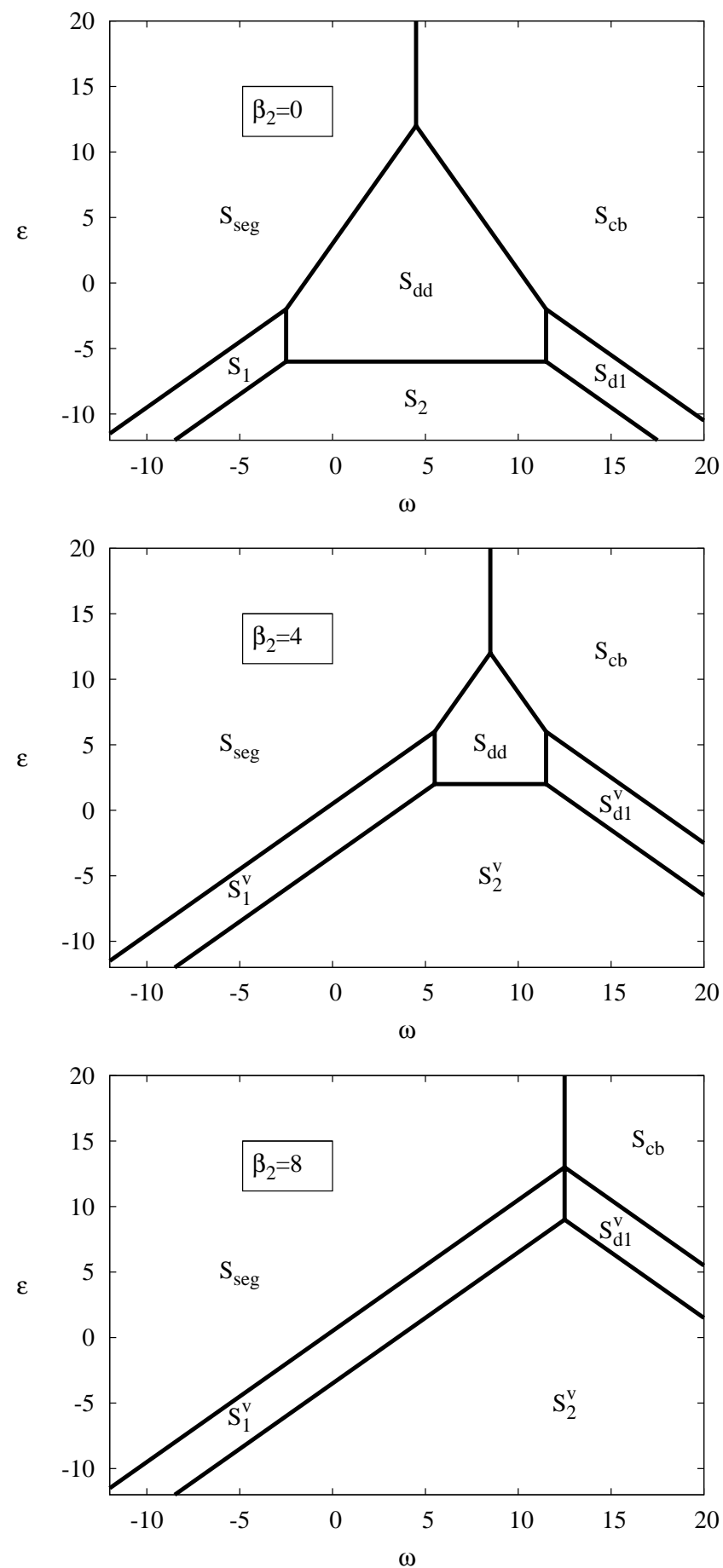

Figure 1: Ground-state phase diagrams in the case of the hole-particle invariant system, according to the effective interaction (4). The representative ion configurations of the displayed phases are shown in Fig. 2. The superscript $v$ by the symbols of phases means that only phases with vertically oriented configurations of ions are stable. $\mathcal{S}_{\text {seg }}$ stands for the segregated phase, which is a mixture of two uniform phases: $\mathcal{S}_{+}$(where $s_{x}=1$ at every site $x)$ and $\mathcal{S}_{-}$(where $s_{x}=-1$ at every site $\left.x\right)$. 


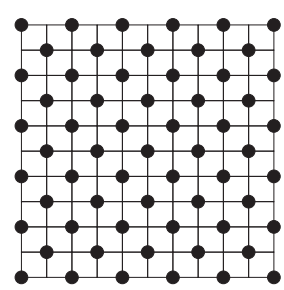

$S_{c b}$

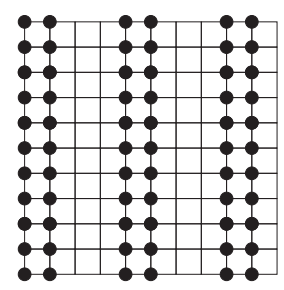

$S_{1}$

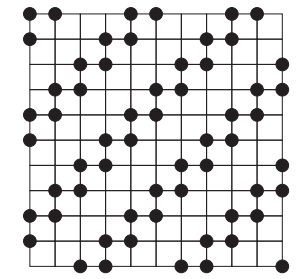

$S_{d d}$

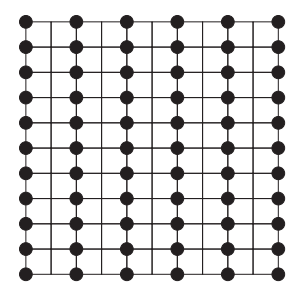

$S_{2}$

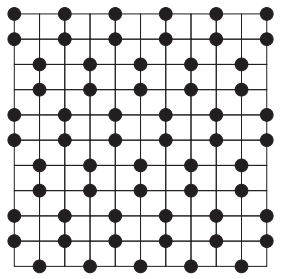

$\boldsymbol{S}_{d l}$

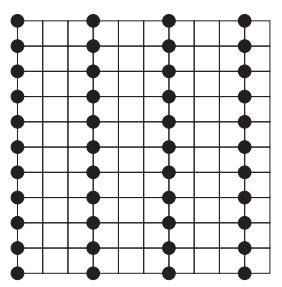

$S_{3}$

Figure 2: Representative ion configurations of the phases considered in the paper. $\mathcal{S}_{c b}$ and $\mathcal{S}_{d 1}$ are examples of checkerboard-like crystals, $\mathcal{S}_{d d}$ - of diagonal-stripe phases, $\mathcal{S}_{1}, \mathcal{S}_{2}, \mathcal{S}_{3}-$ of axial-stripe phases. $\mathcal{S}_{3}$ is absent in the phase diagrams of Fig. 1.

phases $\mathcal{S}_{+}$and $\mathcal{S}_{-}$. If, on the other hand, $\varepsilon$ does not increase, then whether the segregated phase is attained or not depends on the rate of its variation as compared with that of $\omega$. In particular, if $\varepsilon$ decreases sufficiently fast, then the process of deterioration of the crystal terminates in one of the axial-stripe phases $\mathcal{S}_{1}, \mathcal{S}_{1}^{v}, \mathcal{S}_{2}, \mathcal{S}_{2}^{v}$. The state of segregation is not attained. This is reminiscent of the results of qualitative analyses of classical lattice gases with competing interactions, repulsive long-range dipole-dipole or Coulomb interactions and attractive short-range interactions [44, 45, 46].

Now, concerning the sequence of intermediate phases visited by the system before the terminal state is attained, we can see in Fig. 1] only a few elements of this sequence (see the remark above concerning the coexistence lines). Apparently, two cases can be realized. In the first one, the only intermediate phase is a mixture of $\mathcal{S}_{c b}, \mathcal{S}_{+}$, and $\mathcal{S}_{-}$. This occurs, for instance, if the value of $\varepsilon$ in the initial state is large enough and is kept constant. In this case, the system being initially in a crystalline state attains the terminal segregated phase directly via a 1st order phase transition [47, 13. In the second case, before a terminal state (the segregated phase or an axial-stripe phase) is attained, the system visits numerous intermediate phases; at the end of this sequence one always finds axial-stripe phases. This case is realized, for instance, if $\varepsilon$ is sufficiently small and kept constant. Then, the n.n.n., 4th order, effective interaction becomes repulsive and frustrates the interactions stabilizing the checkerboard phase. As a result, as $\omega$ decreases, first the ions form dimers which are distributed in a checkerboard-like manner in the phase $S_{d 1}$, then the the particles form extended strings, i.e. completely filled lattice lines, like in the axial-stripe phases, $S_{1}$ and $S_{3}$. The latter succession of phases is reminiscent of those observed in many other systems with competing interactions (see for instance [44, 48] for results of computer simulations and [21] for rigorous results) and conforms to the following observation: purely repulsive interactions tend to proliferate as small as possible aggregates of particles and arrange them in checkerboard-like crystalline patterns, while attractive interactions tend to make the aggregates of particles as large as possible, which results eventually in segregated-like phases 
with mesoscopic regions completely filled with particles or completely empty. Let us emphasize again that, when a transition from the checkerboard crystal to a segregated phase or a striped phase is accomplished, the sequence of visited phases is, most probably, much longer. The phases visible in the diagrams of Fig. 1, being a part of this sequence, point out only to some tendencies. Apparently, in a process of deterioration of a crystal, striped phase constitute a stage just preceding a transition to a segregated phase.

Finally, let us consider an impact of electron hopping anisotropy on the phase diagrams. For definiteness, we set the vertical hopping weaker than the horizontal one (i.e. $\beta_{2}>0$ ). Any hopping anisotropy breaks the rotational symmetry of the system and out of the phases that are not invariant with respect to all rotations, select some with a specified orientation. For instance, among the axial-stripe phases it stabilizes those stripes that are oriented in the direction of a weaker hopping (vertical in our case). This effect is clearly visible in the phase diagrams of Fig. 1, where for a nonzero anisotropy of n.n. hopping only vertically oriented striped phases remain stable. Let us emphasize that this property holds not only for a truncated effective interaction for which the phase diagrams are actually constructed but for the full quantum system as well. In [49], it was demonstrated that an analogous effect occurs also for diagonal-stripe phases in the presence of an anisotropy of n.n.n. electron hoppings.

The effect of orienting axial-stripe phases in the direction of a weaker hopping has been found also in studies of a Hubbard model in the framework of real-space Hartree-Fock approximation [6].

\section{Specific properties of axial-stripe phases}

All the conclusions of the previous section are based on a rigorous analysis of ground-state phase diagrams of our system, for sufficiently large electron-ion coupling and for a half-filled system, with the main result referring to the stability of striped phases. In this section, we look into properties of the axial-stripe phases to see how important are the above conditions for the drawn conclusions, whether the effect of orienting axial stripes in the direction of weaker hopping, seen in the diagrams of previous section, might persist beyond the regimes of strong coupling and half-filling. Moreover, it should be interesting to unveil the electronic properties of axial-stripe phases, which have been left untouched by the analysis of previous section.

Numerical calculations of the thermodynamic functions considered below are based on exact diagonalization of the electron subsystem in axial striped phases $\mathcal{S}_{1}, \mathcal{S}_{2}$, and $\mathcal{S}_{3}$ (the corresponding spectra and some of their properties are reproduced in Appendix). In this section we present only some properties, verified for the phases $\mathcal{S}_{1}, \mathcal{S}_{2}$, and $\mathcal{S}_{3}$ (see Fig. 20, the lack of subindex "v" indicates that we consider both the horizontally and vertically oriented stripes). We think that the presented properties are characteristic for the whole family of the axial-stripe phases. To support our conclusions, we display only plots of discussed quantities for the phase $\mathcal{S}_{3}$ (see Fig. 2), which is not present in the displayed here holeparticle symmetric phase diagrams (it is visible in diagrams off the hole-particle symmetry [13, 43]), but appears to be a good representative of the family of axial-stripe phases.

We start with comparing the ground-state internal-energy densities of vertically and horizontally oriented axial-stripe phases of a half-filled system, for a small and a large electronhopping anisotropy, see Fig. 3. Let us recall that according to the phase diagrams displayed in previous section, it is the phase whose stripes are oriented in the direction of weaker hopping (vertical in our case) that has a lower energy. This effect occurs in a situation, where all the phases in the phase diagrams constructed with the method used in our work 
(strong-coupling expansion of half-filled system) are insulating. The reason is that for $U$ so large that the expansion is convergent, a gap opens at the Fermi level of any stable periodic phase [27].
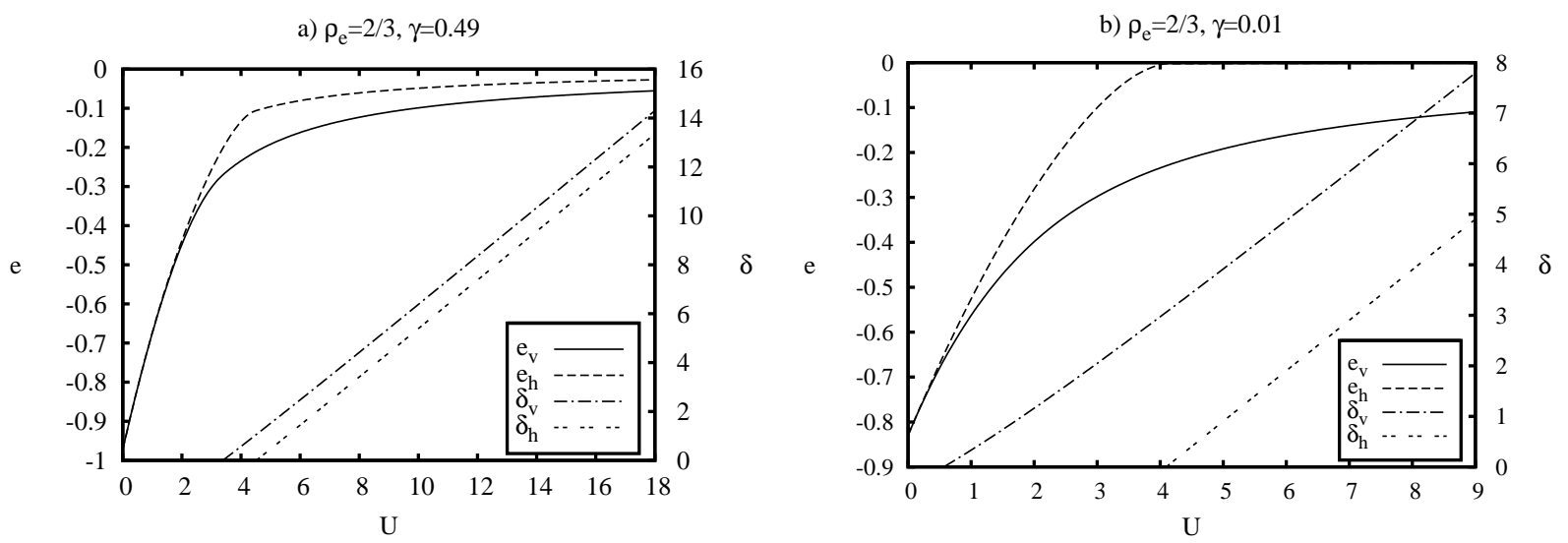

Figure 3: Energy density (per particle), e, and the width, $\delta$, of the gap at the Fermi level in the half-filled phases $\mathcal{S}_{3}^{v}$ and $\mathcal{S}_{3}^{h}$, as functions of $U$, for two values of the anisotropy parameter $\gamma$.

Now, according to our calculations carried out for axial stripes, the vertically oriented stripes seem to have lower energy than those perpendicular to this direction, for all values of $U>0$. Of course, we cannot exclude numerically that this holds only above some positive value of $U$, but in any case this value is much smaller than the values of $U$ admissible in the expansions referred to in Section 2. It follows from dispersion relations (reproduced in Appendix) that for sufficiently large $U$, in both systems, of vertical and horizontal stripes, there is a gap at the Fermi level. This gap opens for smaller values of $U$ and is larger for vertical stripes (those along the direction of weaker hopping), than the horizontal ones. But the relative stability of vertical stripes persists even for the values of $U$, for which there is no gap at the Fermi level. The above observations shine more light on the stability of stripes oriented in the direction of weaker hopping, relative to perpendicular stripes: the effect found in the strong-coupling phase diagrams, when the discussed phases are insulating, may persist also when they are metallic.

However, if we abandon the condition of half-filling the situation is different. In Fig. 4 we display the plots of the ground-state internal-energy densities of vertical and horizontal stripes, for a small and a large electron hopping anisotropy, and for electron densities $\rho_{e}=1 / 2$ and $\rho_{e}=1 / 3$. In the cases displayed in Fig. $4 \mathrm{a}, \mathrm{b}, \mathrm{c}$ there is no gap at the Fermi level, whether the stripes are vertical or horizontal, while in the case of Fig. 4d the gap at the Fermi level opens for vertical stripes (because of sufficiently strong hopping anisotropy). Nevertheless, in all the cases there is a critical value of $U$ above which it is the horizontally oriented phase that has lower energy. That is, for large $U$ but away of half-filling, the vertical stripes exchange their stability with horizontal stripes.

Some of the spatial correlations properties of the electron subsystem are determined by off-diagonal matrix elements (in position basis $\{|\mathbf{r}\rangle\}$ ) of the one-body reduced density operator $\hat{\rho}^{(1)}$ (hereafter called the correlation functions), which can be expressed by the eigenfunctions in position representation. If the Fermi level is located at an upper edge of a band, which does not overlap with the bands of higher energy, then in the limit of an infinite system, the correlation function depending on two lattice positions $\mathbf{r}$ and $\mathbf{r}^{\prime}$ assumes 

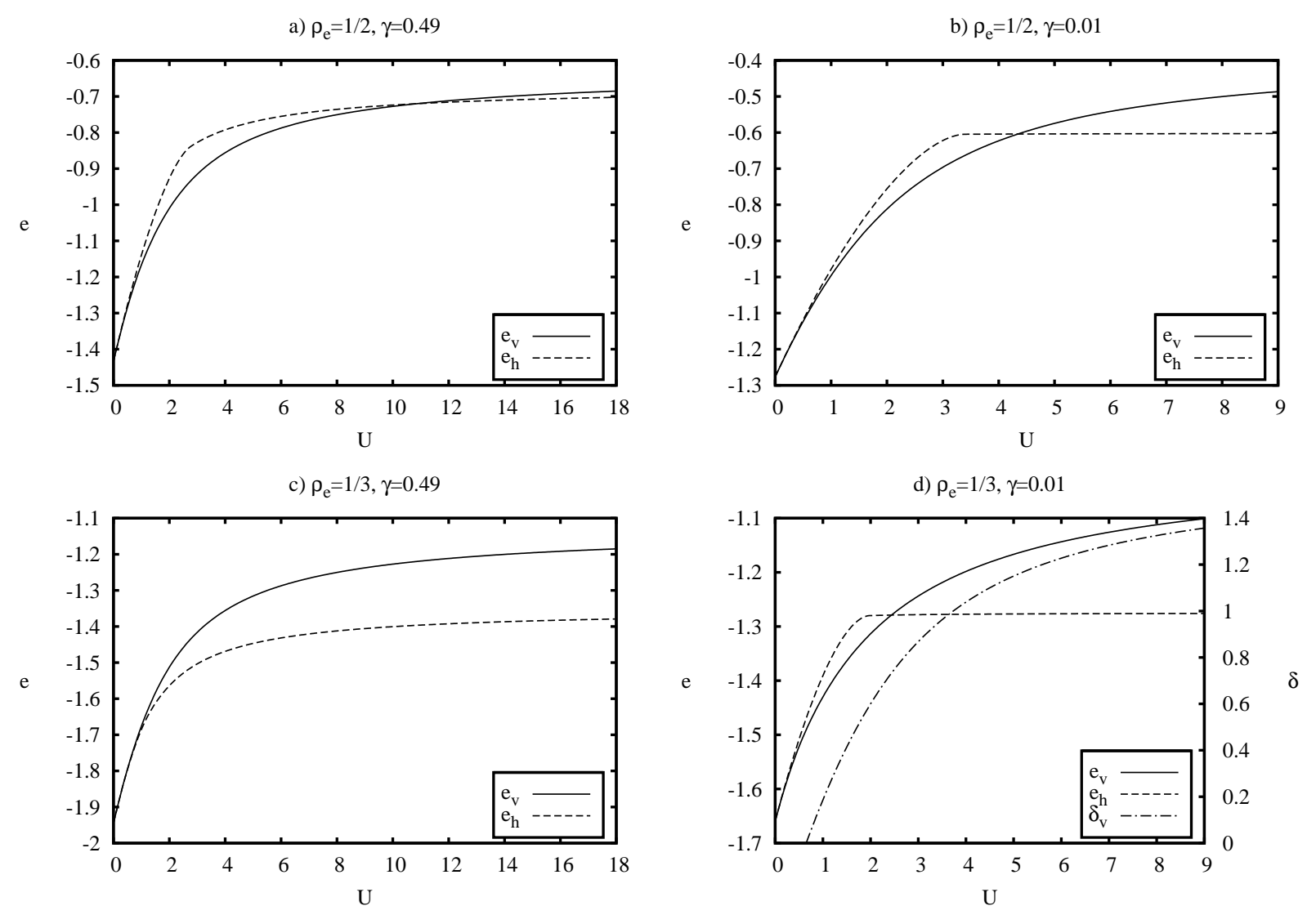

Figure 4: Energy density (per particle), e, in the phases $\mathcal{S}_{3}^{v}$ and $\mathcal{S}_{3}^{h}$ for two values of electron density off the half-filling, and for two values of the anisotropy parameter $\gamma$. Only in case d) a gap of width $\delta$ opens at the Fermi level of $\mathcal{S}_{3}^{v}$.

the form

$$
\left\langle\mathbf{r}\left|\hat{\rho}^{(1)}\right| \mathbf{r}^{\prime}\right\rangle=\frac{1}{(2 \pi)^{2}} \int_{-\pi}^{\pi} d k_{h} \int_{-\pi}^{\pi} d k_{v} K(\mathbf{r}, \mathbf{k}) K^{*}\left(\mathbf{r}^{\prime}, \mathbf{k}\right) \exp \left(i \mathbf{k}\left(\mathbf{r}-\mathbf{r}^{\prime}\right)\right)
$$

where the function $K(\mathbf{r}, \mathbf{k})$ is given in terms of the components (in the plane-wave basis) of eigenvectors corresponding to completely filled bands. As a matter of fact, in the case of axial-stripe phases these components, hence the function $K(\mathbf{r}, \mathbf{k})$, do not depend on the wave vector component in the direction of stripes (see Appendix). Let us consider for definiteness the case of vertically oriented stripes. Then, in the vertical direction, that is for $\mathbf{r}^{\prime}=\mathbf{r}+m \mathbf{j}$, with $m$ being an integer and $\mathbf{j}$ standing for a lattice translation vector in the vertical direction, the correlation function can be written as

$$
\frac{1}{(2 \pi)^{2}} \int_{-\pi}^{\pi} d k_{h} K\left(\mathbf{r}, k_{h}\right) K^{*}\left(\mathbf{r}^{\prime}, k_{h}\right) \int_{-\pi}^{\pi} d k_{v} \exp \left(i m k_{v}\right)
$$

where the last integral vanishes identically.

Let us consider now half-filled axial-stripe phases for fixed $U$. A sufficiently strong anisotropy (a sufficiently weak hopping along the stripes) opens a gap at the Fermi level, 
and then the chains perpendicular to the stripes become independent. Therefore, for sufficiently large anisotropy the ground-state extensive thermodynamic quantities of the whole $2 \mathrm{D}$ system become equal to those of $1 \mathrm{D}$ chains perpendicular to the stripes (i.e. with zero hopping in the direction of stripes). This effect is clearly visible in Fig. 5 a, where for the half-filled, vertically oriented, axial-stripe phase $\mathcal{S}_{3}^{v}$ we show the plots of ground-state internal energy densities versus $U$, for different anisotropies. Starting from sufficiently large $U$,
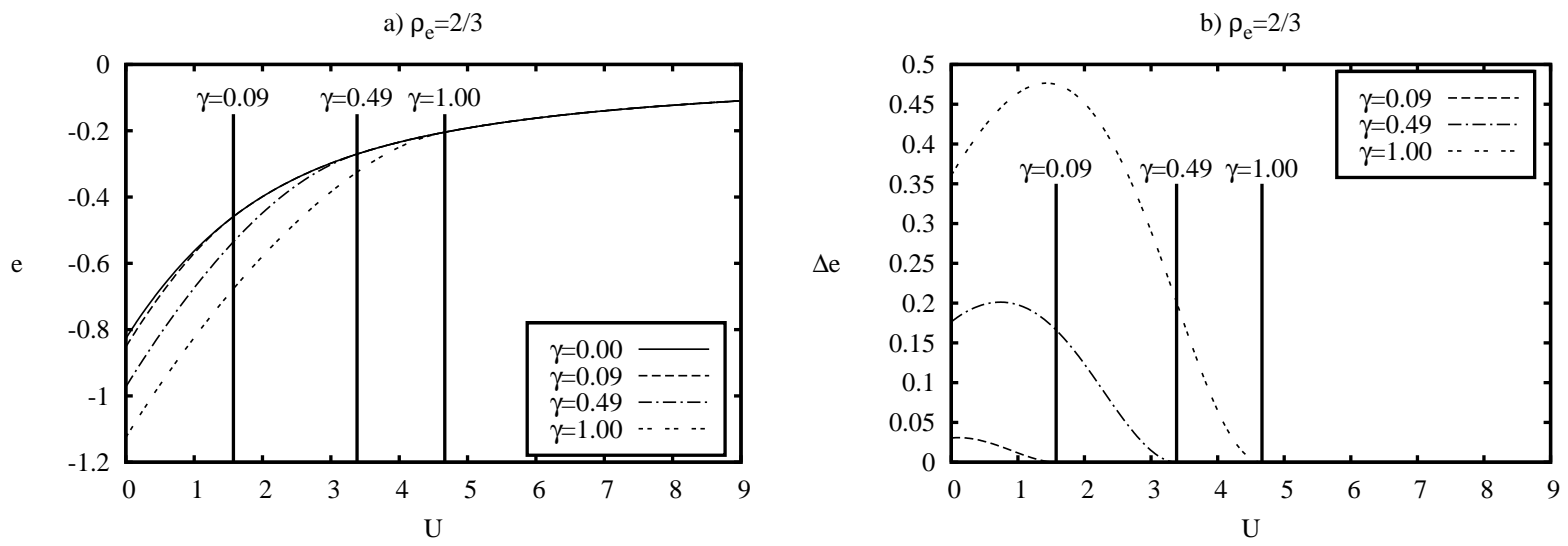

Figure 5: Energy density (per particle), $e$, and relative energy density, $\Delta e=\mid(e(\gamma)-$ $e(0)) / e(0) \mid$, for three nonzero values of the anisotropy parameter $\gamma$, in the phase $\mathcal{S}_{3}^{v}$. The vertical lines are located at the values of $U$, where the gap opens. The highest (continuous) curve in part a) corresponds to the $1 \mathrm{D}$ case.

each plot of a $2 \mathrm{D}$ system coincides with the plot for the corresponding $1 \mathrm{D}$ system. To see more clearly that the plots merge exactly at the value of $U$ for which the gap at the Fermi level opens, we have plotted also the relative differences of internal-energy densities $\Delta e$ (see Fig. 5b and its caption).

It is quite clear that thermal fluctuations will destroy the ideal independence of chains at zero temperature. However, it is interesting to learn how effective they are, by calculating, for instance, the Helmholtz free-energy-density relative differences, $\Delta f$, versus temperature $T$ (see Fig. 6a,b and its caption). We display the corresponding plots for three different anisotropies and two values of $U$. Let us note that the larger is $U$ and the stronger is the anisotropy the larger is the gap at the Fermi level in stripes oriented in the direction of the weaker hopping. For the smaller of the $U$ values (Fig. 6a), the gap opens only for sufficiently strong anisotropy. In the isotropic case (the highest curve) there is no gap, the chains perpendicular to the stripes are dependent even at $T=0$, so the plot of the corresponding $\Delta f$ starts above zero. For the larger of the $U$ values (Fig. 6b) there is a gap for any anisotropy and all the curves start at zero. It is apparent that for a specified tolerance for the deviation $\Delta f$ (of a few per cent of the maximal value), the larger is the gap the higher is the temperature above which this tolerance is exceeded. Below this temperature the chains can be considered as approximately independent.

\section{Summary}

We have studied an extension of a microscopic quantum model of crystallization, proposed by Kennedy and Lieb, in which light hopping electrons interact on-site only with heavy immobile ions. The original model has been extended by a weak short-range attractive interaction 

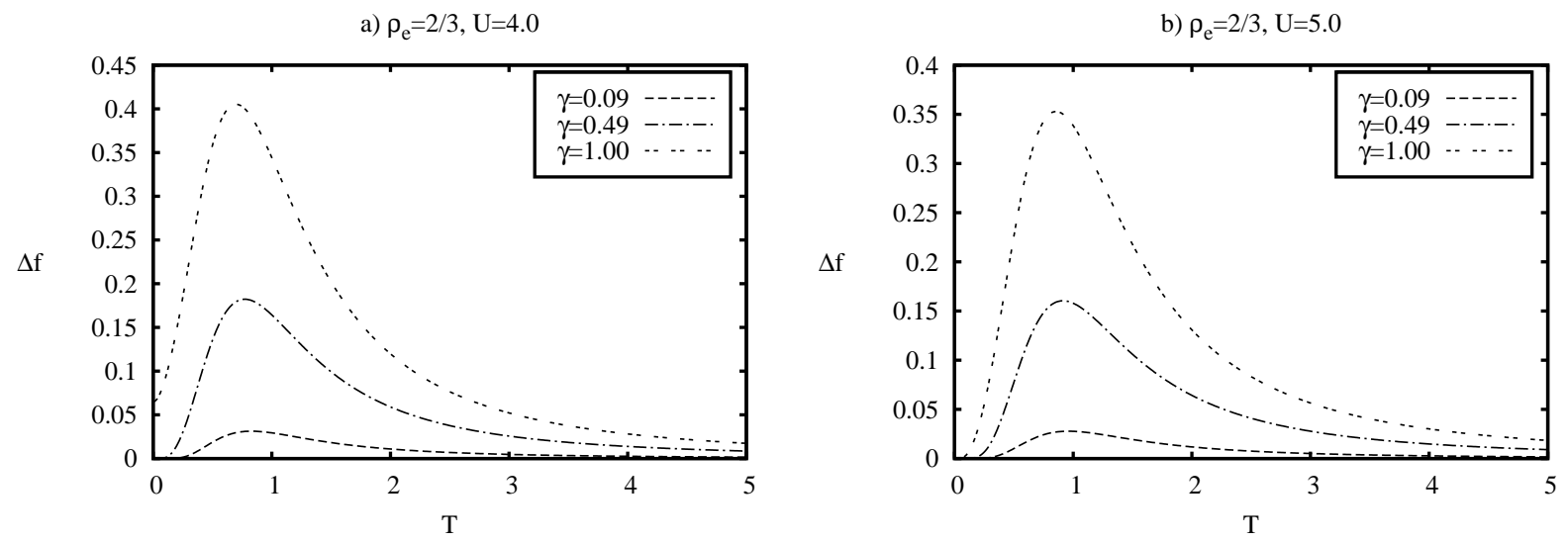

Figure 6: Relative difference of free energy densities, $\Delta f=|(f(\gamma)-f(0)) / f(0)|$, for three values of the anisotropy parameter $\gamma$, in the phase $\mathcal{S}_{3}^{v}$ with two values of $U$.

between ions, whose purpose is to mimic the effect of van der Waals forces. In the framework of the extended model, the stability of striped phases has been analyzed on a rigorous basis. Moreover, we have looked closely into properties of a special class of striped phases, the axial-stripe phases, particularly into properties of their electron subsystem under presence of a hopping anisotropy. It is known already that an anisotropy of the electron hopping has a strong impact on the properties of electron systems [50]; even a half-filled system of free electrons in a constant potential (no gap at the Fermi level) turns into insulator, in the direction of a weaker hopping, as soon as a hopping anisotropy is "switched on". This is a consequence of an exponential decay of correlations in this direction, for any nonzero anisotropy. In the model considered here, the effects of hopping anisotropy are even more striking. We have demonstrated that for sufficiently large anisotropy (depending on the value of $U$ ), the stable axial stripes (i.e. oriented in the direction of a weaker hopping) decouple into 1D chains, which are perpendicular to the stripes. The reason is that (for a given $U)$ a sufficiently strong anisotropy opens a gap at the Fermi level, and this in turn leads to vanishing of electron correlations along the stripes. Consequently, while the ion subsystem develops a 2D long-range order, the electron subsystem may develop only a 1D long-range order, and the compound 2D system behaves like a collection of chains. It is tempting to suggest that an analogous effect may occur in many other systems developing stripes, observed experimentally or studied theoretically, some of which were mentioned in the Introduction. The appearance of stripes in some degrees of freedom may signal a significant reduction of correlations along the stripes among other degrees of freedom of a compound system.

\section{Appendix}

Here we present some spectral quantities for electrons in periodic potentials given by vertical ion configurations $\mathcal{S}_{2}^{v}, \mathcal{S}_{3}^{v}$ and $\mathcal{S}_{1}^{v}$, under periodic boundary conditions. We denote the dispersion relations as $\lambda_{m}^{(l)}(\mathbf{k})$ and the gaps widths as $\delta_{m}^{(l)}$, where $m$ labels configurations $(m=1,2,3)$ and $l$ counts the bands and gaps from bottom to top. The corresponding quantities for horizontal configurations can be obtained by exchanging $t_{v}$ and $t_{h}$ in the formulae below. 
First, for all the considered cases we define $\varepsilon_{v}$ as

$$
\varepsilon_{v}=2 t_{v} \cos \left(k_{v}\right)
$$

Then, for $\mathcal{S}_{2}^{v}$

$$
\begin{aligned}
\lambda_{2}^{(l)}(\mathbf{k}) & =\varepsilon_{v}+u \pm \Delta_{h}, \\
\delta_{2} & =|U|-4\left|t_{v}\right|,
\end{aligned}
$$

where $\varepsilon_{h}=2 t_{h} \cos \left(k_{h} / 2\right), u=U / 2$ and $\Delta_{h}=\sqrt{\varepsilon_{h}^{2}+u^{2}}$;

for $\mathcal{S}_{3}^{v}$

$$
\begin{aligned}
\lambda_{3}^{(l)}(\mathbf{k})= & \varepsilon_{v}+u+2 \sqrt{t_{h}^{2}+u^{2}} \cos \left(\frac{\phi_{h}}{3}+\frac{2 \pi l}{3}\right) \\
& l=1,2,3 ; \\
\delta_{3}^{(l)}= & -4\left|t_{v}\right|+2 \sqrt{3\left(t_{h}^{2}+u^{2}\right)} \sin \left(\frac{\phi_{h}^{*}}{3}+\frac{2 \pi(l-1)}{3}\right), \\
& l=1,2
\end{aligned}
$$

where $u=U / 3, \phi_{h}=\arccos \left(\frac{u^{3}+t_{h}^{3} \cos \left(k_{h}\right)}{\left(t_{h}^{2}+u^{2}\right)^{3 / 2}}\right)$ and $\phi_{h}^{*}=\arccos \left(\frac{u^{3}+(-1)^{l-1}\left|t_{h}^{3}\right|}{\left[u^{2}+t_{h}^{2}\right]^{3 / 2}}\right)$;

for $\mathcal{S}_{1}^{v}$

$$
\begin{aligned}
\lambda_{1}^{(l)}(\mathbf{k}) & =\varepsilon_{v}+u \pm \sqrt{2 t_{h}^{2}+u^{2} \pm 2\left|t_{h}\right| \Delta_{h}}, \\
\delta_{1}^{(1)} & =-4\left|t_{v}\right|+\sqrt{t_{h}^{2}+\left(\left|t_{h}\right|+|u|\right)^{2}}-\sqrt{t_{h}^{2}+\left(\left|t_{h}\right|-|u|\right)^{2}}, \\
\delta_{1}^{(2)} & =-4\left|t_{v}\right|+2\left(\sqrt{t_{h}^{2}+u^{2}}-\left|t_{h}\right|\right), \\
\delta_{1}^{(3)} & =\delta_{1}^{(1)}
\end{aligned}
$$

where $u=U / 2, \Delta_{h}=\sqrt{t_{h}^{2} \cos ^{2}\left(k_{h} / 2\right)+u^{2}}$.

It might be helpful to represent the above formulae graphically. We have chosen to plot the band edges separated by a gap and the Fermi levels as functions of $U$. In Figs. 7 and 8, these plots are displayed for ion configurations $\mathcal{S}_{3}^{v}$ and $\mathcal{S}_{3}^{h}$, in all the cases considered in previous section. Let $\left\{|\mathbf{k}\rangle_{i}\right\}$ be a plane-wave basis of the space of single electron states, labelled by wave-vectors $\mathbf{k}$ and index $i=1, \ldots, l$ enumerating the sublattices of the underlying periodic ion configuration. Let $h_{\mathbf{k}}$ be the matrix, in the plane-wave basis, of the Hamiltonian of a single electron in an external field specified by an axial-stripe configuration of horizontal period $l$ (which amounts to the number of sublattices). The components of this matrix are labelled by the sublattices of the underlying periodic ion configuration. The matrix has the following structure:

$$
h_{\mathbf{k}}=\left(\begin{array}{cccc}
\varepsilon_{v}+C_{1} & f_{1,2}\left(k_{h}\right) & \ldots & f_{1, l}\left(k_{h}\right) \\
f_{2,1}\left(k_{h}\right) & \varepsilon_{v}+C_{2} & \ldots & f_{2, l}\left(k_{h}\right) \\
\ldots & \ldots & \ddots & \ldots \\
f_{l, 1}\left(k_{h}\right) & f_{l, 2}\left(k_{h}\right) & \ldots & \varepsilon_{v}+C_{l}
\end{array}\right),
$$

where the matrix elements $f_{i, j}\left(k_{h}\right)$ are some functions of wave vector component $k_{h}$, and $C_{i}$ are independent of the wave vector. In this matrix, the only elements which depend on wave 

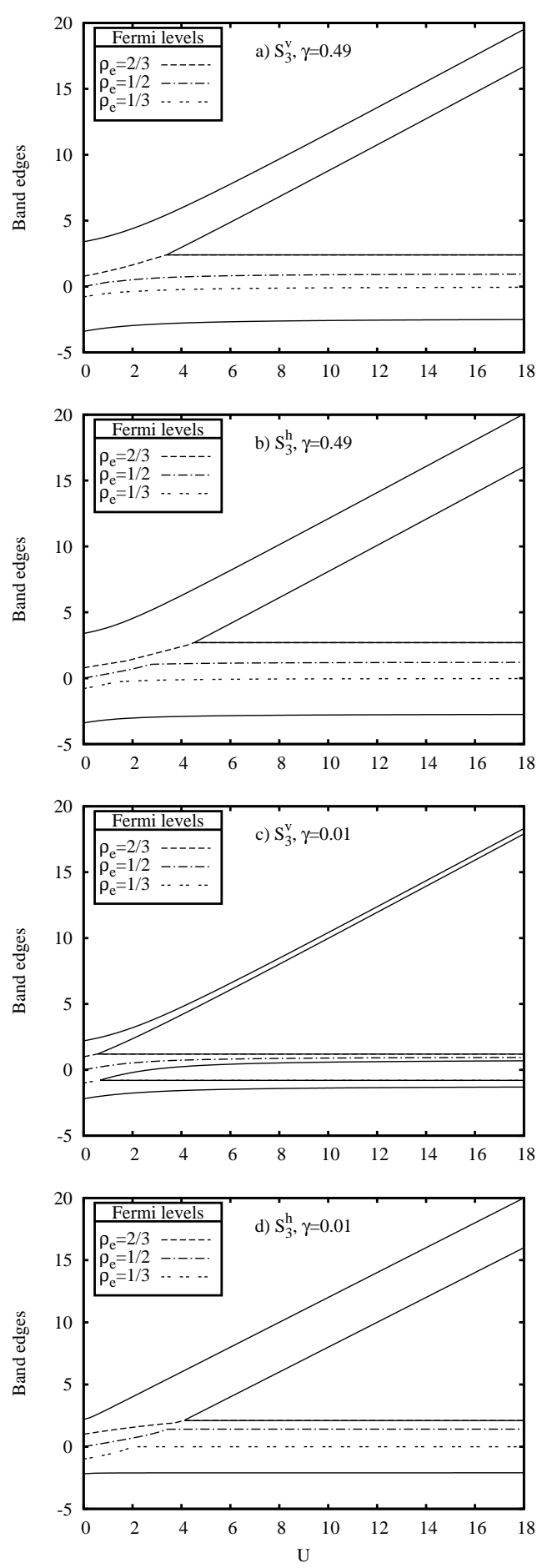

Figure 7: Band edges and Fermi levels for configurations $\mathcal{S}_{3}^{v}$ and $\mathcal{S}_{3}^{h}$. Solid lines represent the band edges that are separated by a gap (note that in cases a), b) and d) there is no gap between the first and the second band) while dashed, dash-dotted and dotted lines the Fermi levels. When a line of a Fermi level joins an intersection of two solid lines, then it should be continued along the lower of the solid lines. These plots correspond to the cases shown in Fig. 3 and Fig. 4 .
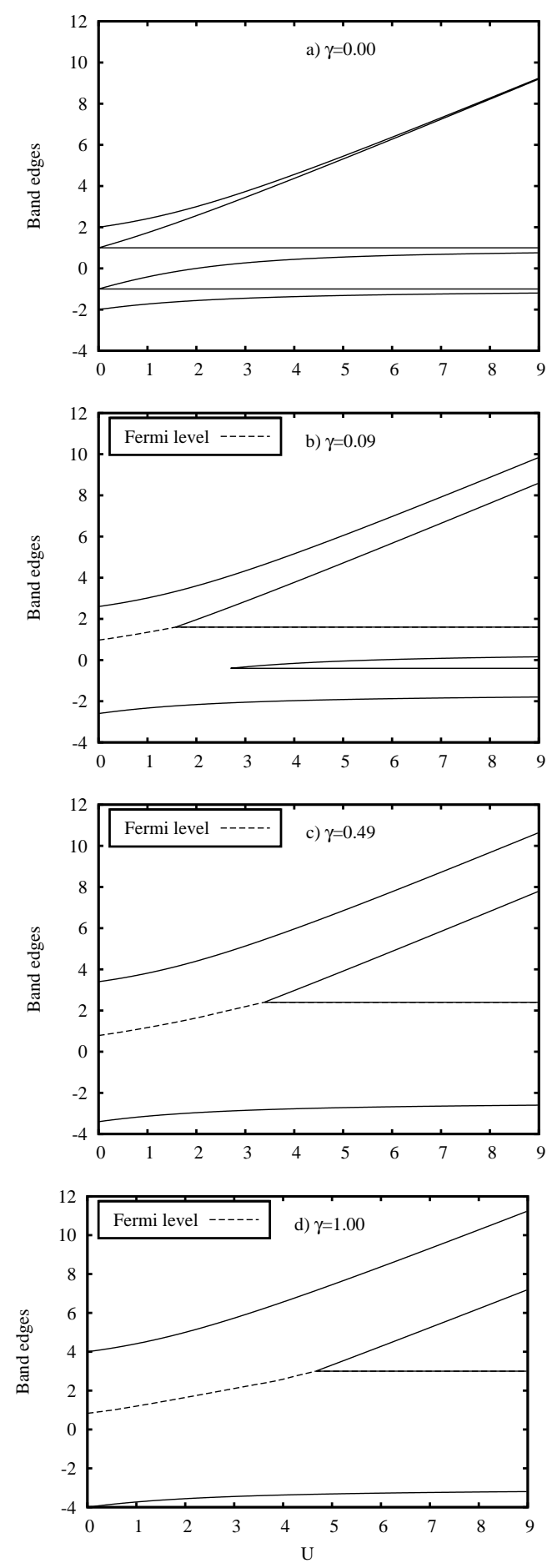

Figure 8: Band edges and Fermi levels for configuration $\mathcal{S}_{3}^{v}$ with electron density $\rho_{e}=2 / 3$, Solid lines represent the band edges (note that in cases c) and d) there is no gap between the first and the second band) and the dashed line - the Fermi level. When a line of a Fermi level joins an intersection of two solid lines, then it should be continued along the lower of the solid lines. In the case a) the Fermi-level line coincides with the upper edge of the second band. These plots correspond to the cases shown in Fig. 5 . 
vector component $k_{v}$ are the diagonal elements, $\left(h_{\mathbf{k}}\right)_{i i}=\varepsilon_{v}+C_{i}$. Then, the solutions of the characteristic equation assume the form: $\lambda(\mathbf{k})=\varepsilon_{v}+f\left(k_{h}\right)$, with $f$ being some function of the horizontal component of $\mathbf{k}$. Consequently, the equations for eigenvectors $\alpha$ with components

$\alpha_{i}, \sum_{i}\left(h_{\mathbf{k}}-\lambda(\mathbf{k})\right)_{i j} \alpha_{j}=0$, and so their solutions, do not depend on the vertical component of $\mathbf{k}$.

\section{References}

[1] K. Kern, H. Niehus, A. Schatz, P. Zeppenfeld, J. Goerge, and G. Comsa, Phys. Rev. Lett. 67, 855 (1991)

[2] R. Allenspach and A. Bischof, Phys. Rev. Lett. 69, 3385 (1992)

[3] M. Seul and R. Wolfe, Phys. Rev. Lett. 68, 2460 (1992)

[4] D. Poilblanc and T. M. Rice, Phys. Rev. B 39, 9749 (1989); J. Zaanen and O. Gunnarsson, Phys. Rev. B 40, 7391 (1989)

[5] K. Machida, Physica C 158, 192 (1989); M. Kato, K. Machida, H. Nakanishi, and M. Fujita, J. Phys. Soc. Jpn. 59, 1047 (1990)

[6] A. M. Oleś, Acta Physica Polonica B 31, 2963 (2000)

[7] S. R. White and D. J. Scalapino, Phys. Rev. Lett. 80, 1272 (1998)

[8] S. R. White and D. J. Scalapino, Phys. Rev. Lett. 81, 3227 (1998)

[9] A. W. Sandvik, S. Daul, R. R. P. Singh, and D. J. Scalapino, Phys. Rev. Lett. 89, $247201(2002)$

[10] N. G. Zhang and C. L. Henley, Phys. Rev. B 68, 014506 (2003)

[11] C. L. Henley and N.-G. Zhang, Phys. Rev. B 63, 233107 (2001)

[12] R. Lemański, J. K. Freericks, and G. Banach, Phys. Rev. Lett. 89, 196403 (2002)

[13] V. Derzhko, J. Jȩdrzejewski, Physica A 349, 511 (2005)

[14] K. Sasaki, Surf. Sci. 318, L1230 (1994)

[15] U. Löw, V.J. Emery, K. Fabricius, and S.A. Kivelson, Phys. Rev. Lett. 72, 1918 (1994)

[16] I. Booth, A. B. MacIsaac, J. P. Whitehead, K. De'Bell, Phys. Rev. Lett. 75, 950 (1995)

[17] J. Arlett, J. P. Whitehead, A. B. MacIsaac, K. De’Bell, Phys. Rev. B 54, 3394 (1996)

[18] A. D. Stoycheva and S. J. Singer, Phys. Rev. Lett. 84, 4657 (2000)

[19] D. Valdez-Balderas and D. Stroud, Phys. Rev. B 72, 214501 (2005)

[20] Z. Nussinov, arXiv:cond-matt/0105253

[21] A. Giuliani, J. L. Lebowitz, and E. H. Lieb, Phys. Rev. B 74, 064420 (2006); A. Giuliani, J. L. Lebowitz, and E. H. Lieb, Phys. Rev. B 76, 184426 (2007); A. Giuliani, J. L. Lebowitz, and E. H. Lieb, arXiv:0811.3078 
[22] J. M. Tranquada, D. J. Buttrey, V. Sachan, and J. E. Lorenzo, Phys. Rev. Lett. 73, 1003 (1994)

[23] J. M. Tranquada, B. J. Sternlieb, J. D. Axe, Y. Nakamura and S. Uchida, Nature (London) 375, 561 (1995)

[24] T. Kennedy, E. H. Lieb, Physica A 138, 320 (1986)

[25] L. M. Falicov and J. C. Kimball, Phys. Rev. Lett. 22, 997 (1969)

[26] U. Brandt, R. Schmidt, Z. Phys. B 63, 45 (1986)

[27] C. Gruber and N. Macris, Helv. Phys. Acta 69, 850 (1996)

[28] J. Jȩdrzejewski and R. Lemański, Acta Phys. Pol. B 32, 3243 (2001)

[29] T. Kennedy, Rev. Math. Phys. 6, 901 (1994)

[30] A. Messager and S. Miracle-Solé, Rev. Math. Phys. 8, 271 (1996)

[31] C. Gruber, N. Macris, A. Messager and D. Ueltschi, J. Stat. Phys. 86, 57 (1997)

[32] N. Datta, R. Fernández and J. Fröhlich, J. Stat. Phys. 96, 545 (1999)

[33] T. Kennedy, J. Stat. Phys. 91, 829 (1998)

[34] K. Haller, Commun. Math. Phys. 210, 703 (2000)

[35] K. Haller and T. Kennedy, J. Stat. Phys. 102, 15 (2001)

[36] C. Gruber, D. Ueltschi and J. Jȩdrzejewski, J. Stat. Phys. 76, 125 (1994)

[37] Z. Gajek, J. Jȩdrzejewski, and R. Lemański, Physica A 223, 175 (1996)

[38] G. I. Watson and R. Lemański, J. Phys. Cond. Matt. 7, 9521 (1995)

[39] P. Farkasovsky, Eur. Phys. J. B 20, 209 (2001)

[40] P. Lemberger, J. Phys. A 25, 715 (1992)

[41] J. K. Freericks, E. H. Lieb, and D. Ueltschi, Phys. Rev. Lett. 88, 106401 (2002)

[42] J. K. Freericks, E. H. Lieb and D. Ueltschi, Commun. Math. Phys. 227, 243 (2002)

[43] V. Derzhko and J. Jȩdrzejewski, J. Stat. Phys. 126, 467 (2007)

[44] I. Booth, A. B. MacIsaac, J. P. Whitehead, K. De'Bell, Phys. Rev. Lett. 75, 950 (1995)

[45] R. Jamei, S. Kivelson, and B. Spivak, Phys. Rev. Lett. 94, 056805 (2005)

[46] B. Spivak and S. A. Kivelson, Phys. Rev. B 70, 155114 (2004)

[47] V. Derzhko and J. Jȩdrzejewski, Physica A 328, 449 (2003)

[48] D. Valdez-Balderas and D. Stroud, Phys. Rev. B 72, 214501 (2005)

[49] V. Derzhko, J. Phys. A 39, 11145 (2006)

[50] J. Jȩdrzejewski and T. Krokhmalskii, Europhys. Lett. 78, 37002 (2007) 